\title{
Factors influencing the development of otitis media with effusion in children
}

\author{
Katarzyna Pazdro-Zastawny, Tomasz Zatoński, Krystyna Orendorz-Frączkowska, \\ Karolina Dorobisz, Monika Morawska-Kochman \\ Clinic of Otolaryngology Head and Neck Surgery, Wroclaw Medical University, Wroclaw, Poland
}

\section{ABSTRACT}

Otitis media with effusion (OME) is one of the most frequent childhood diseases and the most common cause of hearing loss in childhood. Eighty per cent of all children have an episode of OME by the age of 10 years, typically by the age of three years. The disease is characterised by an accumulation of fluid in the middle ear behind an intact tympanic membrane in the absence of signs and symptoms of acute infection. This causes progressive conductive hearing loss, which is the main symptom of the disease. When left untreated, OME may lead to the development of adhesive otitis media, tympanic membrane abnormalities, tympanic sclerosis, cholesterol granuloma, or acquired primary cholesteatoma. The aetiology of OME is multifactorial. Allergy is considered an important aetiological mechanism of OME. An association between allergy, proinflammatory and immunoregulatory cytokines, obesity, a high-fat diet, and OME is discussed in this paper.

\section{KEY WORDS:}

obesity, children, allergy, otitis media with effusion.

\section{INTRODUCTION}

Otitis media with effusion (OME) is one of the most common clinical conditions in children and a major cause of hearing loss in childhood. The vast majority of preschool children experience at least one episode of OME, and about $90 \%$ of children under the age of two have had OME. The prevalence of the disease decreases with age, from $20 \%$ at the age of two years to $8 \%$ at the age of eight years [1].

OME is characterised by an accumulation of effusion in the middle ear (ME) behind an intact tympanic membrane in the absence of signs and symptoms of acute infection such as otorrhoea, otalgia, or fever. Fluid accumulation leads to progressive conductive hearing loss, which is the main symptom of the disease. When left untreated,
OME may lead to the development of retraction pockets, tympanic sclerosis, cholesterol granuloma, or acquired primary cholesteatoma [2].

The aetiology of OME is multifactorial. An immature function of the immune system and a dysfunction of the eustachian tube (ET), often due to adenoid hypertrophy causing mechanical obstruction in the nasopharynx or allergy, are the most important aetiological factors. Predisposing factors of OME include: day-care attendance, older siblings, male sex, artificial feeding, low socioeconomic status, exposure to tobacco smoke (very often connected with allergy or atopy), and recurrent upper respiratory tract and ME infections [1]. Seasonal variation of OME has been observed. Gordon et al. [3] revealed that patients who developed the OME at intervals with the onset in autumn were connected with poor prognosis

\section{ADDRESS FOR CORRESPONDENCE:}

Katarzyna Pazdro-Zastawny, Clinic of Otolaryngology Head and Neck Surgery, Wroclaw Medical

University, 213 Borowska St., 50-556 Wroclaw, Poland, ORCID: 0000-0001-8056-1198,

e-mail: kasiapz@poczta.fm 
in terms of the resolution of ME effusion, which implies that clinicians should consider taking a more conservative therapeutic approach to OME management in the spring and summer, and a more aggressive treatment in the fall and winter months.

OME may result in long-term sequelae, such as learning disabilities or impaired linguistic, social, or emotional development [4]. A previous case of effusion in the ME has a negative impact on the vestibular part of the inner ear [5].

\section{THE ROLE OF ALLERGY IN OTITIS MEDIA WITH EFFUSION}

There is evidence suggesting epidemiological and pathophysiological links between allergy and OME [6]. Its incidence in OME ranges from $0 \%$ to $80 \%$ [7-9].

A recent study [10] revealed higher prevalence of atopy or allergy in children with a recurrent presence of episodes of OME between acute infections (atopy: 73.0\% vs. $39.5 \%, p<0.001$; allergy: $60.0 \%$ vs. $36.1 \%, p=0.049$ ), which may suggest that children with recurrent acute otitis media (AOM) with OME are clinically different from children with simple recurrent $\mathrm{AOM}$, because they have a more complex clinical presentation that includes not only adenoidal disease and audiological impairment, but also an underlying allergy or atopy.

The role of allergy in the aetiopathogenesis of OME may be one of the following mechanisms: 1) the ME reacting as a shock organ, 2) a dysfunction of the ET secondary to an allergic inflammatory swelling or the ET acting as a shock organ, 3) an inflammatory nose obturation, or 4) aspiration of bacteria-laden allergic nasopharyngeal secretion into the ME cavity $[9,11]$. The latter three mechanisms are associated with ET dysfunction.

ME mucosa and the rest of the upper respiratory tract epithelium evolve from the same ectoderm and, similarly to the mucosa of the upper and lower respiratory tract, are capable of an immune response $[9,12]$.

Studies of OME patients revealed that this group of patients have higher incidence of atopic conditions such as allergic rhinitis (AR), eczema, and asthma [12]. The immunopathological mechanism underlying the development of OME in patients with allergy is largely attributed to the effects of Th2 cytokines and their receptors $[9,12]$. According to Nguyen, allergic inflammatory changes among children with atopy are not limited to the ME mucosa, but also include the nasopharyngeal cavity. Therefore, the ME may behave in a "similar manner to the lungs under allergic inflammatory insults", and the "ME may be included in the united airways". The study suggests that, in addition to the general inflammatory changes in the respiratory tract, the ME is capable of producing a much stronger local inflammatory reaction [12]. IgE-mediated allergy has long been considered a key factor in about one-third of recurrent OME patients based on clinical observations and skin testing [8].
One of the main factors responsible for the development and persistence of inflammation in the ME is ET dysfunction resulting from AR [6]. According to Quaranta et al., chronic rhinitis is present in more than $70 \%$ of children with AR, and $60 \%$ of them had OME [13].

\section{FOOD ALLERGY}

The connection between food allergy (FA) and OME has been investigated in several studies $[14,15]$. According to Caffarelli et al. [16], FA may be one of the key factors in the development of OME, especially in children under two years of age. Juntti et al. revealed that children who had an allergy to cow's milk in infancy, even in properly treated cases, experienced significantly more recurrent episodes of otitis media (OM). In their study a higher proportion of children with cows' milk allergy, who had developed respiratory atopy, had had recurrent OM ( $27 \%$ vs. $12 \%, p=0.009$ ), and had undergone adenoidectomy and or tympanostomy, compared with the controls ( $48 \%$ vs. $28 \%, p=0.005$ ). Asthma and/or allergic rhinitis and atopic dermatitis increased the risk for OME [17]. The possibility of FA should be considered in all paediatric patients with recurrent, serious $\mathrm{OM}$ and a search made for the suspected food allergen in order to determine the proper diagnostic and therapeutic intervention. According to Arroyave [18], food allergens associated with recurrent OME included milk, egg, beans, citrus, and tomato. An elimination diet resulted in a significant amelioration of the effusion in children with OME and FA. A challenge diet with the suspected offending food induced a recurrence of the OME. Therefore, all patients with recurrent OME should be investigated for FA.

The middle and inner ear are immunologically responsive and also susceptible to food hypersensitivities. Chronic OME can improve the following treatment of food allergies. Patients with FA may not respond to conventional treatments. In chronic inflammatory disorders involving the upper aerodigestive tract, FA must be considered in the differential diagnosis [19].

\section{THE ROLE OF CYTOKINES IN THE DEVELOPMENT OF OTITIS MEDIA WITH EFFUSION}

Cytokines participate in the activation, proliferation, chemotaxis, immunoregulation, growth, differentiation, and apoptosis of cells. Pro-inflammatory and immunoregulatory cytokines are involved in ME inflammatory response [20].

Numerous studies confirm that many pro-inflammatory cytokines (e.g. TNF- $\alpha$, IL-1 $\beta$, IL-6, IL-8) are thought to play a critical role as mediators in OME. They participate in the development and maintenance of inflammation in the ME cleft. Moreover, cytokines act in the network of mutual hierarchic interactions. The primary 
pro-inflammatory cytokines IL- $1 \beta$ and TNF- $\alpha$, during OME, stimulate production of secondary cytokines, including interleukin-6 (IL-6) [21-26].

Zielnik-Jurkiewicz et al. revealed elevated levels of TNF- $\alpha$, IL-1 $\beta$, IL- 6 , and IL- 8 in the effusions in all children with chronic OME, with the highest levels observed in non-atopic children. Children with atopy had higher concentration of IL-1 $\beta$. In children without atopy, IL- $1 \beta$ and TNF- $\alpha$ levels correlated with the age and the number of past acute OM episodes. According to the authors, this supports the idea of including anti-inflammatory management in the treatment of OME, regardless of atopy [26].

Himi et al. [23] showed that cytokines, especially IL- $1 \beta$, contribute to infiltration into the ME by inflammatory cells. This implies that the persistent presence of cytokines in ME effusion could be a factor in prolonged OME. It promotes fibroblast activation and proliferation, and growth of osteoclasts in the ME tissues. Thus, this cytokine leads to fibrosis and affects bone tissue, and is therefore associated with the maintaining chronic inflammation [21].

IL-6 is considered to be an important cytokine in the pathogenesis of OME and is associated with the early stages of OME. IL-6 is a pleiotropic cytokine exerting multidirectional effects on the cells of both innate and acquired immune systems. It is recognised as a key factor regulating the defence mechanisms. IL- 6 has the ability to initiate and regulate acute inflammation as well as to facilitate and direct an acquired immune response. It also exerts systemic effects. It activates B cells in ME inflammation. Increased and/or prolonged production of IL-6 facilitates progression of the immune/inflammatory response from an acute into a chronic one. It participates in a bone remodelling process leading to tympanosclerosis [21]. More elevated levels of IL-6 in ME effusion are also observed in children with more profound hearing loss [23].

OME is defined as chronic when ME effusion has been present for at least three months. IL-8 is considered to play a crucial role in the long duration of the disease and regulates production of mucous. IL- 8 is responsible for enhancement of the recruitment of neutrophils into the ME cleft, release of lysosomal enzymes, and cell injury. Pospiech et al. [27] evaluated the concentration of IL-8 in ME effusions. Their study revealed significantly elevated levels of IL-8 in children with chronic, long-lasting OME (mean 12 months) in comparison to that in acute purulent otitis, and significantly higher than that in serous chronic OM. Also, increased levels of IL-8 correlated with an early recurrence of OME. According to the authors, it supports the idea that IL- 8 is responsible for the chronicity and duration of inflammatory process in ME.

Age was observed to have a significant effect on the levels of specific cytokines. Elevated levels of IL-6 have been found in younger children [23].

\section{OBESITY AND OTITIS MEDIA WITH EFFUSION}

An association between obesity [28-30], a high-fat diet (HFD), and OME has been reported [31]. Obese children are more likely to experience OM than normal-weight children, and they have increased chances of recurrent OM. Socioeconomic factors, a history of breastfeeding, and the presence of an allergic disorder or chronic adenoid/tonsil disorder did not affect the association between obesity and OM [32].

A healthy diet can protect children against obesity. Tapiainen et al. [33], in a cross-sectional study on 1006 children attending child day care centres, revealed that dietary factors such as daily intake of carbohydrates could be associated with the occurrence of OM. An increased intake of fruit and berries could be protective against AOM (regression coefficient $-0.51,95 \%$ CI -0.98 to $-0.03, p=0.04)$.

Kim et al. [34] evaluated the difference between a study group, consisting of 155 children aged two to seven years, with OME, who underwent unilateral or bilateral ventilation tube insertion, and control groups in BMI and serum triglyceride and total cholesterol concentrations, and the difference between the obese and non-obese subgroups in the frequency of ventilation tube insertion. It was revealed that mean BMI $(p=0.01)$ and mean TC levels $(p=0.04)$ were significantly higher in the study group than in the control group. In obese children the incidence of OME, while high, was not associated with the frequency of ME drainage. Childhood obesity was significantly higher in children with OME. This finding suggests that childhood obesity could have an effect on the development of OME.

Obesity may contribute to the development of OME through the following mechanisms: 1) by inducing changes in the levels of cytokines, 2) by altering host immunity, 3) by causing gastroesophageal reflux, and 4) by changing the proper structure and function of ET through an accumulation of fat around the ET and the nasopharynx [35].

Adipose tissue is considered to be an endocrine organ. It affects the intrinsic endocrine system and inflammatory paths and therefore interferes with other organs. Adipose tissue is immunologically active. It is known that allergic inflammation is promoted by cytokines and interleukins.

Changes in the ME mucosa and an accumulation of adipose tissue in patients with active cellular responses, persistent inflammation in the ME, or hyper-secretion of ME effusion lead to an elevated quantity of IL- 6 and TNF- $\alpha$ [36]. Weight gain results in an increase of levels of IL- 6 and TNF- $\alpha$. The presence of IL- 6 in the ME effusion was identified in the majority of patients with chronic OME. The IL- 6 amount correlated with TNF- $\alpha$ concentration [37].

A HFD induces changes in immunomodulatory function by modifying T-cell responses and the production of 
IFN- $\gamma$; increasing the concentration of leptin may result in an increased frequency of upper respiratory tract infections and ET dysfunction [38].

A HFD decreases the production of Th1-type cytokines and affects the function of macrophages and $\mathrm{T}$ cells [39]. A HFD may influence T-cell proliferation and differentiation, and this is connected with the type of fatty acid. This suggests that the type of fatty acid in the diet, rather than its quantity, determines the effect of an immune reaction in a certain individual [40].

A HFD reduces the expression of Toll-like receptor 2 (TLR2). An impaired TLR2 response following a HFD might be partially responsible for an increased risk of infection in HFD-induced obesity [41].

Laryngopharyngeal reflux (LPR) usually observed in obesity may be another cause of OME [42-44]. Abtahi et al. [44] showed a higher prevalence of gastroesophageal reflux in children with OM and controls (58\% and 22\%, respectively, $p=0.0005)$. Among children with recurrent acute $\mathrm{OM}$ and $\mathrm{OME}$, its prevalence was significantly higher compared to controls (61.1\% vs. $22 \%$ for AOM, $p=0.004$, and $72.7 \%$ vs. $22 \%, p=0.003$ ). The authors suggest that looking for gastroesophageal reflux in children with OM may help improve treatment outcomes.

Pepsin and pepsinogen in ME effusion are probably caused by LPR and may be involved in the pathogenesis of OME [45]. It was revealed that the levels of pepsinogen protein expressed in cytoplasm of epithelial cells and clearance under epithelial cells in adenoid specimens from children with OME were significantly higher than in children without OME. The concentrations of pepsin in dry ears were significantly lower than those in serous and mucus ears in the OME group $(p<0.001)$. Finally, the concentration of pepsinogen in ME effusion was positively correlated with the expression intensity of pepsinogen protein in cytoplasm of epithelial cells $(p<0.05)$ in the OME group. Also, LPR is aggravated by a HFD, through increased intragastric pressure and the transdiaphragmatic gastroesophageal pressure gradient [46].

Obesity may predispose to OME also in a mechanical way. Depositions of the adipose tissue surround ET and its opening, which lead to its obstruction [47].

Altered taste sensation has been reported in chronic OME patients. Recurrent episodes of OM or chronic inflammatory process in the ME in the course of OME interfere with taste by altering taste function, which is associated with the progressive chorda tympani branch of cranial nerve VII dysfunction [31]. A change in the sense of taste also provokes OME patients to consume more food or liquids in order to accomplish the same strength of taste as non-OME controls [35].

The change in taste sensitivity might result in a preference for a HFD [48]. In a study by Choi the BMI in children with OME was significantly higher than in healthy controls $(p=0.02)$. The anterior part of the tongue had a significantly higher taste threshold in the OME than in the control group (anterior right, $p=0.03$; anterior left, $p=0.04$ ), and chemical taste test results showed that sweet and salty tastes were significantly lower in the OME group (sweet, $p=0.02$; salty, $p=0.04$ ) [49].

On the other hand, a HFD may predispose to OME. A diet rich in fat increases the risk of OME in children. HFD interferes with the immunomodulatory system, resulting in OME by inducing higher susceptibility for ET dysfunction and upper respiratory tract infections. Choi et al. [29] suggest that a HFD has a close connection with OME, instead of obesity mediating a HFD and OME. Kaya et al. [30] state that the prevalence of overweight or obesity is higher in children with chronic OME compared to healthy controls. However, current evidence cannot determine whether overweight or obesity is a risk factor for developing chronic OME, or the opposite is the case.

\section{CONCLUSIONS}

The aetiology of OME is multifactorial. Allergy is considered to be an important aetiological mechanism of OME because it probably leads to ET dysfunction. Paediatric obesity may have an effect on the development of OME because the two produce similar immunological alterations. The exact underlying mechanism by which obesity predisposes to OME needs to be elucidated in clinical studies.

\section{DISCLOSURE}

The authors declare no conflict of interest.

\section{REFERENCES}

1. Zernotti ME, Pawankar R, Ansotegui I, et al. Otitis media with effusion and atopy: is there a causal relationship? World Allergy Organ J 201714; 10: 37.

2. Ryding M, White P, Kalm O. Course and long-term outcome of 'refractory' secretory otitis media. J Laryngol Otol 2005; 119: 113-118.

3. Gordon MA, Grunstein E, Burton WB. The effect of the season on otitis media with effusion resolution rates in the New York Metropolitan area. Int J Pediatr Otorhinolaryngol 2004; 68: 191-195.

4. Augustsson I, Engstrand I. Hearing loss as a sequel of secretory and acute otitis media as reflected by audiometric screening of Swedish conscripts. Int J Pediatr Otorhinolaryngol 2006; 70: 703-710.

5. Pazdro-Zastawny K, Pośpiech L, Zatoński T. Long-term evaluation of the effect of middle ear effusion on the vestibular system in children. Int J Pediatr Otorhinolaryngol 2018; 109: 13-16.

6. Wasowka-Królikowska K, Dynowski J, Godzisz J. The way of nutrition and frequency of otitis media in hospitalized infants and 3-year-old children. Pol Merkur Lekarski 1998; 5: 333-334.

7. Black N. Causes of glue ear. An historical review of theories and evidence. J Laryngol Otol 1985; 99: 953-966.

8. Bernstein JM, Doyle WJ. Role of IgE-mediated hypersensitivity in otitis media with effusion: pathophysiologic considerations. Ann Otol Rhinol Laryngol Suppl 1994; 163: 15-19.

9. Sobol SE, Taha R, Schloss MD, et al. T(H)2 cytokine expression in atopic children with otitis media with effusion. J Allergy Clin Immunol 2002; 110: 125-130. 
10. Torretta S, Pignataro L, Carioli D, et al. Phenotype Profiling and Allergy in Otitis-Prone Children. Front Pediatr 2018; 6: 383.

11. Chantzi FM, Kafetzis DA, Bairamis T, et al. IgE sensitization, respiratory allergy symptoms, and heritability independently increase the risk of otitis media with effusion. Allergy 2006; 61: 332-336.

12. Nguyen LH, Manoukian JJ, Sobol SE, et al. Similar allergic inflammation in the middle ear and the upper airway: evidence linking otitis media with effusion to the united airways concept. J Allergy Clin Immunol 2004; 114: 1110-1115.

13. Bernstein JM. Role of allergy in eustachian tube blockage and otitis media with effusion: a review. Otolaryngol Head Neck Surg 1996; 114: 562-568.

14. Quaranta N, Milella C, Iannuzzi L, Gelardi M. A study of the role of different forms of chronic rhinitis in the development of otitis media with effusion in children affected by adenoid hypertrophy. Int J Pediatr Otorhinolaryngol 2013; 77: 1980-1983.

15. Aydogan B, Kiroglu M, Altintas D, et al. The role of food allergy in otitis media with effusion. Otolaryngol Head Neck Surg 2004; 130: 747-750.

16. Caffarelli C, Savini E, Giordano S, et al. Atopy in children with otitis media with effusion. Clin Exp Allergy 1998; 28: 591-596.

17. Juntti $\mathrm{H}$, Tikkanen S, Kokkonen J, et al. Cow's milk allergy is associated with recurrent otitis media during childhood. Acta Otolaryngol 1999; 119: 867-873.

18. Arroyave CM. Reccurent otitis media with effusion and food allergy in pediatric patients. Rev Alergol Mex 2001; 48: 141-144.

19. Ramakrishnan JB. The role of food allergy in otolaryngology disorders. Curr Opin Otolaryngol Head Neck Surg 2010; 18: 195-199.

20. Tilg H, Moschen AR. Adipocytokines: mediators linking adipose tissue, inflammation and immunity. Nat Rev Immunol 2006; 6 : 772-783.

21. Smirnova MG, Kiselev SL, Gnuchev NV, et al. Role of the pro-inflammatory cytokines tumor necrosis factor-alpha, interleukin-1 beta, interleukin- 6 and interleukin- 8 in the pathogenesis of the otitis media witheffusion. Eur Cytokine Netw 2002; 13: 161-172.

22. Smirnova MG, Kiselev SL, Gnuchev NV, et al. The role of food allergy in otolaryngology disorders. Curr Opin Otolaryngol Head Neck Surg 2010; 18: 195-199.

23. Himi T, Suzuki T, Kodama H, et al. Immunologic characteristics of cytokines in otitis media with effusion. Ann Otol Rhinol Laryngol Suppl 1992; 157: 21-25.

24. Russo E, Smith CW, Friedman EM, et al. Cell adhesion molecules and cytokines in middle ear effusions in children with or without recent acute otitis media. Otolaryngol Head Neck Surg 2004; 130: 242-248.

25. Yellon RF, Leonard G, Marucha PT, et al. Characterization of cytokines present in middle ear effusions. Laryngoscope 1991; 101: 165-169.

26. Zielnik-Jurkiewicz B, Stankiewicz-Szymczak. Pro-inflammatory interleukins in middle ear effusions from atopic and non-atopic children with chronic otitis media with effusion. Eur Arch Otorhinolaryngol 2016; 273: 1369-1378.

27. Pospiech L, Jaworska M, Kubacka M. Soluble L-selectin and interleukin-8 in otitis media with effusion. Auris Nasus Larynx 2000; 27: 213-217.

28. Kim SH, Park DC, Byun JY, et al. The relationship between overweight and otitis media with effusion in children. Int J Obes (Lond) 2011; 35: 279-282.

29. Choi HG, Sim S, Kim SY, Lee HJ. A high-fat diet is associated with otitis media with effusion. Int J Pediatr Otorhinolaryngol 2015; 79: 2327-2331.

30. Kaya S, Selimoğlu E, Cureoğlu S, Selimoğlu MA. Relationship between chronic otitis media with effusion and overweight or obesity in children. J Laryngol Otol 2017; 131: 866-870.
31. Peracchio HL, Henebery KE, Sharafi M, et al. Otitis media exposure associates with dietary preference and adiposity: a community-based observational study of at-risk preschoolers. Physiol Behav 2012; 106: 264-271.

32. Kuhle S, Kirk SF, Ohinmaa A, et al. The association between childhood overweight and obesity and otitis media. Pediatr Obes 2012; 7: 151-157.

33. Tapiainen T, Paalanne N, Arkkola T, et al. Diet as a risk factor for pneumococcal carriage and otitis media: a cross-sectional study among children in day care centers. PLoS One 2014; 9: e90585.

34. Kim JB, Park DC, Cha CI, Yeo SG. Relationship between pediatric obesity and otitis media with effusion. Arch Otolaryngol Head Neck Surg 2007; 133: 379-382.

35. Kim YJ, Cha SH, Lee HY, et al. Decreased pattern-recognition receptor-mediated cytokine mRNA expression in obese children with otitis media with effusion. Clin Exp Otorhinolaryngol 2014; 7: 7-12.

36. Bixler EO, Vgontzas AN, Lin HM, et al. Sleep disordered breathing in children in a general population sample: prevalence and risk factors. Sleep 2009; 32: 731-736.

37. Cottam DR, Mattar SG, Barinas-Mitchell E, et al. The chronic inflammatory hypothesis for the morbidity associated with morbid obesity: implications and effects of weight loss. Obes Surg 2004; 14: 589-600.

38. Wallace FA, Miles EA, Evans C, et al. Dietary fatty acids influence the production of Th1- but not Th2-type cytokines. J Leukoc Biol 2001; 69: 449-457.

39. de Jong AJ, Kloppenburg M, Toes RE, Ioan-Facsinay A. Fatty acids, lipid mediators, and T-cell function. Front Immunol 2014; 5: 483.

40. Adler BJ, Green DE, Pagnotti GM, et al. High fat diet rapidly suppresses B lymphopoiesis by disrupting the supportive capacity of the bone marrow niche. PLoS One 2014; 9: e90639.

41. Wan Z, Durrer C, Mah D, et al. One-week high-fat diet leads to reduced toll-like receptor 2 expression and function in young healthy men. Nutr Res 2014; 34: 1045-1051.

42. Abdel-aziz MM, El-Fattah AM, Abdalla AF. Clinical evaluation of pepsin for laryngopharyngeal reflux in children with otitis media with effusion. Int J Pediatr Otorhinolaryngol 2013; 77: 1765-1770.

43. Al-Saab F, Manoukian JJ, Al-Sabah B, et al. Linking laryngopharyngeal reflux to otitis media with effusion: pepsinogen study of adenoid tissue and middle ear fluid. J Otolaryngol Head Neck Surg 2008; 37: 565-571.

44. Abtahi SH, Kazerooni A, Brejis N, et al. Prevalence and characteristics of gastroesophageal reflux in children with otitis media in Isfahan, Iran. Adv Biomed Res 2016; 5: 81

45. Luo HN, Yang QM, Sheng Y, et al. Role of pepsin and pepsinogen: linking laryngopharyngeal reflux with otitis media with effusion in children. Laryngoscope 2014; 124: E294-300.

46. Ahmed S, Arjmand E, Sidell D. Role of obesity in otitis media in children. Curr Allergy Asthma Rep 2014; 14: 469.

47. O'Brien LM, Sitha S, Baur LA, Waters KA. Obesity increases the risk for persisting obstructive sleep apnea after treatment in children. Int J Pediatr Otorhinolaryngol 2006; 70: 1555-1560.

48. Bartoshuk LM, Duffy VB, Hayes JE, et al. Psychophysics of sweet and fat perception in obesity: problems, solutions and new perspectives. Philos Trans R Soc Lond B Biol Sci 2006; 361: 1137-1148.

49. Shin IH, Park DC, Kwon C, Yeo SG. Changes in taste function related to obesity and chronic otitis media with effusion. Arch Otolaryngol Head Neck Surg 2011; 137: 242-246. 